\title{
ESTRATEGIAS PARA \\ LA DIFUSIÓN DE LOS \\ ITINERARIOS CULTURALES: \\ EL CAMINO REAL DE \\ TIERRA ADENTRO
}

ROCIO MARCELA ACOSTA CHÁVEZ 


\section{ROCIO MARCELA ACOSTA CHÁVEZ}

Licenciada en ciencias de la comunicación. Maestría en historia del arte urbano. Directora de Laberinto Comunicación, despacho de consultoría en patrimonio cultural. Miembro del equipo de investigación en la elaboración del expediente técnico del Camino Real de Tierra Adentro para su inscripción en la Lista del Patrimonio Mundial. Cestora de sitio del Camino Real de Tierra Adentro ante el Centro de Patrimonio Mundial de la UNESCO. Miembro de número del ICOMOS mexicano y miembro del Comité Científico Internacional de Itinerarios Culturales del ICOMOS. 


\section{RESUMEN \\ ABSTRACT}

El concepto de itinerario cultural es relativamente nuevo, ya que fue aprobado en el año 2008, durante la $16^{\mathrm{a}}$.Asamblea General del ICOMOS, celebrada en Quebec, Canadá. Este concepto es muestra -como lo afirma su preámbulo- de la evolución de las ideas acerca de la visión de este concepto y de la importancia que cobran el entorno y la escala territorial. La conservación de un bien del patrimonio mundial depende en gran medida del grado de conocimiento y apropiación que tenga la comunidad en la que este se sitúa y de cómo se presente al exterior. Esta presentación se deberá hacer siguiendo parámetros de ética y utilizando las herramientas y técnicas adecuadas, para lo cual el ICOMOS ha creado documentos específicos como son las cartas generadas por los distintos comités científicos. El Camino Real de Tierra Adentro, primer itinerario cultural inscrito en la Lista del Patrimonio Mundial en el año 2010, representa un reto en cuanto a su difusión y promoción, dada la complejidad y variedad de los sitios que lo componen.

Palabras clave: patrimonio, itinerarios culturales, presentación, interpretación, turismo, Camino Real de Tierra Adentro.

The concept of cultural route is relatively recent, since the Charter on Cultural Routes was ratified by the 16th Ceneral Assembly of ICOMOS, in Québec (Canada), on October 4, 2008. This concept is proof -as it is exposed in the preamble of the Charter- of the evolution of the ideas around the vision of cultural property and of the increasing importance of their setting and territorial scale.

The conservation of a World Heritage property depends on great measure on the degree of knowledge and appropriation of the community in which this is located, and on how this is presented to the exterior. The presentation of these sites should be made following ethical criteria and using the pertinent tools and techniques, for which the ICOMOS -through its diverse Scientific Committees- has created specific documents known as Charters.

The Camino Real de Tierra Adentro, first cultural route inscribed on the World Heritage List in 2010, represents a challenge as for its diffusion and promotion, given the complexity and variety of the sites that integrate it.

Key Words: heritage, cultural routes, presentation, interpretation, tourism, Camino Real de Tierra Adentro. 


\section{INTRODUCCIÓN}

Podemos afirmar que el concepto de itinerario cultural es relativamente nuevo, ya que fue aprobado en el año

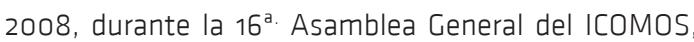
celebrada en Quebec, Canadá.

Este concepto de patrimonio es muestra, según lo dice su preámbulo (ICOMOS, 2008, p. 1), de la evolución de las ideas acerca de la visión de este concepto y de la importancia que cobran el entorno y la escala territorial.

Gracias a estos avances nos es posible ahora comprender y valorar las manifestaciones del patrimonio de esta naturaleza desde una perspectiva multidimensional, dando justo valor a cada elemento dentro de un fenómeno de movilidad humana asociado a una vía de comunicación que fue creada con un fin específico, que además haya tenido una cierta duración y en el cual se hayan dado intercambios culturales recíprocos, de los cuales haya evidencias tangibles e intangibles.

El primer itinerario cultural inscrito en la Lista del Patrimonio Mundial de la UNESCO fue el Camino Real de Tierra Adentro, sitio mexicano que constituye el primer itinerario terrestre trazado por los españoles en América, en el cual se aprecia claramente una serie de bienes tangibles (de entre los cuales se inscribieron 60 sitios) a lo largo de los 1.600 kilómetros inscritos, y que fue un rico entramado de experiencias culturales, sociales, étnicas, científicas, económicas, biológicas, arquitectónicas, artísticas y, por supuesto, humanas.

Dar a conocer este bien, primeramente a autoridades y ciudadanos locales y a nivel nacional, implica una tarea ardua; así como también lo es la presentación del bien ante el mundo y la difusión que se desee hacer con distintos fines.

La conservación de un bien del patrimonio mundial va a depender en buena medida del grado de conocimiento y apropiación que tenga la comunidad en la que este se sitúa y de cómo se presente al exterior, y afortunadamente el ICOMOS dispone de un buen cuerpo de documentos sobre las distintas categorías y conceptos del patrimonio cultural que han sido desarrollados por los comités científicos y se han aprobado en las distintas asambleas con el consenso internacional.

Como se menciona en el preámbulo de la Carta de ICOMOS para la Interpretación y Presentación de Sitios de Patrimonio Cultural: «Desde su fundación, en 1965, como organización internacional de profesionales del sector patrimonial dedicada al estudio, la documentación y la protección de sitios patrimoniales, ICOMOS se ha esforzado en promover la ética de la conservación en todas sus actividades, así como en contribuir a promover la apreciación pública del patrimonio material de la humanidad en todas sus formas y diversidad» (ICOMOS, 2008b, p. 1).

Hay que tener en cuenta que una declaratoria de patrimonio de la humanidad despierta el interés en torno al bien, muchos deseamos conocer el valor universal excepcional que lo hace merecedor de tal distinción, y de igual manera muchos sectores buscarán generar beneficios a las comunidades y a posibles negocios -muchos con fines turísticos-, para los que será necesario realizar una presentación adecuada del bien. 
Lamentablemente, sabemos que la promoción no siempre se realiza con apego a la ética, y muchas veces los sitios son promovidos de una manera errónea, lo cual genera daños a los bienes. De igual manera, si un bien no es conocido por sus habitantes va a ser difícil que se pueda valorar lo que no se conoce, de ahí que sea de gran importancia la realización de campañas que sigan los principios de interpretación y presentación del patrimonio.

Esto fue tema de análisis durante el XXII Symposium Internacional de Conservación del Patrimonio Monumental, en Pátzcuaro, Michoacán, México: «Como resultado de su inscripción en dicha lista, los sitios quedan sujetos a una serie de fenómenos de distinta naturaleza que no siempre significan su preservación. Es por ello que la elaboración de planes y programas de manejo con una visión integral de protección constituye una tarea de especial trascendencia a seguir, previamente y después de la inclusión en la lista» (ICOMOS México, 2002, punto 4). Y precisamente la Carta para la Interpretación y Presentación de Sitios de Patrimonio Cultural es el documento que destaca la importancia de la comunicación adecuada de los itinerarios culturales.

\section{EL CAMINO REAL DE TIERRA ADENTRO}

El Camino Real de Tierra Adentro (CRTA) fue una vía terrestre que se comenzó a trazar de manera formal a raíz de los descubrimientos de minerales en la región septentrional de las Indias. Su primera etapa partía de la ciudad de México, capital del Virreinato de la Nueva
España, hasta el Real de Minas de Nuestra Señora de los Zacatecas (descubierto en 1546). Fue tal la riqueza encontrada en esas tierras y el afán conquistador de los españoles, que la expansión continuó hasta la villa de Santa Fe del Reino del Nuevo México, fundada en 1598 (hoy en el estado de Nuevo México, en Estados Unidos), alcanzando una extensión de más de 2.600 kilómetros.

En este bien «se conservan de manera extraordinaria las evidencias culturales tangibles e intangibles, producto del trabajo de extracción y beneficio de la plata, del intenso comercio de bienes, del dominio militar, del poder civilizador de la evangelización y una formidable estructura administrativa ideada para controlar el inmenso territorio conquistado desde la metrópoli española» (INAH 2009).

EI CRTA se desprende como un ramal del sistema de comunicación más dinámico y abierto Ilamado Camino Real Intercontinental Español. Su escala alcanzó una extraordinaria dimensión territorial, ya que se extendió por más de 2.600 kilómetros y perduró pasados los trescientos años, vinculando de manera directa varias culturas migratorias y autóctonas (la española y la amerindia, principalmente).

Este itinerario comprende 60 sitios de distinta naturaleza distribuidos en 11 entidades de la República Mexicana (el Distrito Federal y los estados de México, Hidalgo, Querétaro, Guanajuato, Jalisco, Aguascalientes, Zacatecas, San Luis Potosí, Durango y Chihuahua), entre los que se encuentran cinco ciudades ya incluidas anteriormente en la lista del patrimonio mundial. Entre las tipologías de los sitios encontramos ciudades, villas 


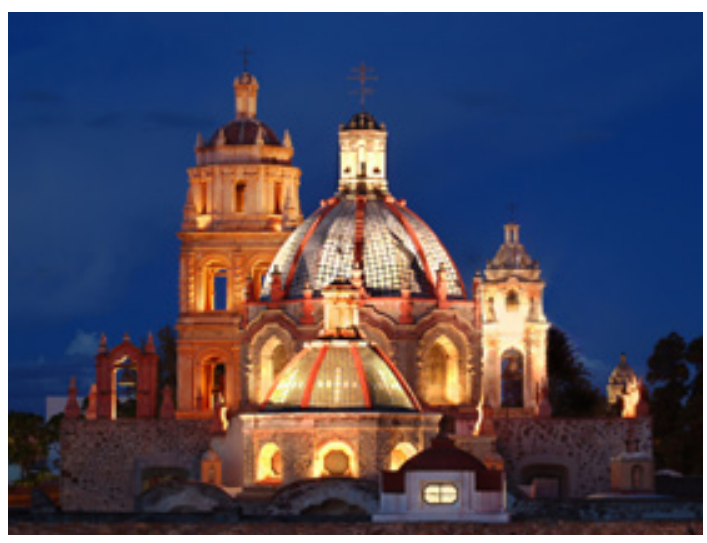

01.

de españoles, reales de minas, haciendas, presidios, colegios religiosos, misiones, conventos, hospitales, cementerios, puentes, tramos del camino real, sitios con pinturas rupestres y elementos representativos del paisaje natural. Esta amplia gama representa un reto en cuanto a su difusión y promoción, dada la complejidad y variedad de los sitios que la componen.

Para los 60 sitios es necesario diseñar estrategias que permitan primeramente dar a conocer el bien entre los habitantes y autoridades del lugar, con el fin de crearles un sentido de propiedad que fomente las acciones de conservación, y más adelante presentar el bien a los visitantes de una manera adecuada.

\section{COMUNICACIÓN, INTERPRETACIÓN, PRESENTACIÓN Y DIFUSIÓN DE LOS ITINERARIOS CULTURALES}

El tema de la comunicación de los bienes culturales ha sido una preocupación constante. La Convención sobre la Protección del Patrimonio Mundial, Cultural y Natural de la UNESCO, aprobada por la Conferencia Ceneral en su decimoséptima reunión celebrada en París, Francia, el 16 de noviembre de 1972, indica claramente en sus artículos 4 y 6.2, que «los estados parte de la Convención se comprometen a identificar, proteger, conservar,
01.

\section{Campanario del Templo \\ de San Francisco.}

San Luis Potosí

FOTO: CORTESÍA SECRETARÍA

DE TURISMO DE SLP rehabilitar y transmitir a las generaciones futuras el patrimonio cultural y natural situado en su territorio» (UNESCO, 1972, pp. 3 у 4).

La Carta para la Interpretación y Presentación de Sitios del Patrimonio Cultural hace referencia a que «[las] primeras cartas del ICOMOS destacan la importancia de la comunicación pública como parte primordial en un proceso de conservación más amplio (describiéndolo como "difusión", “divulgación”, "presentación" e "interpretación"), reconociendo de forma implícita que cada acto de conservación del patrimonio -dentro de todas las tradiciones culturales del mundo- es por su naturaleza un acto comunicativo» (ICOMOS, 2008b).

Varias décadas después, y en respuesta a la evolución de conceptos y categorías del patrimonio cultural, se han presentado y aprobado documentos conocidos como «cartas», que consisten en la definición y principios sobre las categorías del patrimonio, en las cuales se establece la metodología particular para su identificación, investigación y promoción; así como para su protección, conservación, y gestión.

La Carta de Itinerarios Culturales, desarrollada por el Comité Científico Internacional de Itinerarios Culturales del ICOMOS, fue ratificada por la 16 $6^{\mathrm{a}}$. Asamblea General del ICOMOS, en Quebec, Canadá (ICOMOS, 2008, p. 2) 
y nos da la definición de un itinerario cultural: «Toda vía de comunicación terrestre, acuática o de otro tipo, físicamente determinada y caracterizada por poseer su propia y específica dinámica y funcionalidad histórica al servicio de un fin concreto y determinado, que reúna las siguientes condiciones:

- Ser resultado y reflejo de movimientos interactivos de personas, así como de intercambios multidimensionales, continuos y recíprocos de bienes, ideas, conocimientos y valores entre pueblos, países, regiones o continentes, a lo largo de considerables períodos.

- Haber generado una fecundación múltiple y recíproca, en el espacio y en el tiempo, de las culturas afectadas que se manifiesta tanto en su patrimonio tangible como intangible.

- Haber integrado en un sistema dinámico las relaciones históricas y los bienes culturales asociados a su existencia».

Con base en esta definición nos damos cuenta de que estamos hablando de bienes que poseen elementos definitorios, como contexto, contenido, valor de conjunto compartido, carácter dinámico y entorno; que también poseen indicadores específicos, como estructura de la red y su sustrato material, construcciones asociadas a la funcionalidad del recorrido para servir a su finalidad específica, elementos de comunicación, la existencia de manifesta- ciones culturales de origen compartido, como son prácticas, tradiciones, costumbres y usos comunes de carácter religioso, ritual, lingüístico, festivo, culinario, etcétera, influencia recíproca en las artes, la ciencia, la técnica, la tecnología, y los bienes culturales materiales e inmateriales relacionados con la funcionalidad histórica del itinerario.

Hay asimismo diferentes tipos de itinerarios culturales (ICOMOS, 2008, pp. 3 y 4) que pueden clasificarse:

- Atendiendo a su dimensión territorial: local, nacional, regional, continental o intercontinental.

- De acuerdo con su dimensión cultural: dentro de una región cultural determinada o a lo largo de diversas áreas geográficas que hayan compartido o sigan compartiendo un proceso de influencias recíprocas en la formación o evolución de sus valores culturales.

- Por su objetivo o función social, económica, política o cultural. Estas características pueden tener una naturaleza compartida en un contexto multidimensional.

- Por lo que se refiere a su duración temporal: Ios que ya no se utilizan, o los que continúan desarrollándose bajo las influencias de intercambios socioeconómicos, políticos, y culturales.

- Por su configuración estructural: lineal, circular, cruciforme, radial o en red.

- En cuanto a su marco natural: terrestre, acuático, mixto o de otra naturaleza física. 
Para lograr una adecuada difusión del CRTA es necesario tener claro no solamente el concepto de itinerario cultural, sino también poder identificar los elementos definitorios, de manera que cada entidad pueda hacer la difusión interna para los habitantes y autoridades enfocándose en sus sitios pero sin alejarse del valor de conjunto compartido.

De igual manera es importante considerar el vínculo con el entorno y con el patrimonio intangible, así como aclarar el concepto de itinerario cultural para evitar que se confunda con una ruta turística.

El carácter multidimensional de los itinerarios culturales, y en particular la variedad de los sitios que conforman el Camino Real de Tierra Adentro, nos brindan la oportunidad de generar una amplia gama de posibilidades para su difusión y promoción.

Como también lo indica esta carta (ICOMOS, 2008, p. 6) en su punto 6: «La protección, conservación/preservación, promoción y gestión de un itinerario cultural requiere del estímulo de la conciencia social y la participación de los habitantes de las áreas concernidas que comprenda el itinerario», la gestión y la sustentabilidad de un itinerario requieren de procesos comunicativos adecuados para lograr enviar mensajes que ayuden a entender y proteger el bien.

\section{INTERPRETACIÓN Y PRESENTACIÓN}

La interpretación se refiere a todas las actividades realizadas para incrementar la concienciación pública y propiciar un mayor conocimiento del sitio de patrimonio cultural (ICOMOS, 2008b, p. 2). Estas actividades incluyen publicaciones impresas y electrónicas, conferencias, instalaciones sobre el sitio, programas educativos y de formación, actividades comunitarias, investigación y su monitoreo y evaluación.

La presentación se refiere a la comunicación planeada del contenido de interpretación, accesibilidad física e infraestructura de interpretación en los sitios. Se puede transmitir a través de medios como paneles informativos, exposiciones, senderos señalizados, conferencias y visitas guiadas, multimedia y páginas web.

Los objetivos de la presentación e interpretación se enfocan en facilitar la comprensión del bien para se pueda valorar y de esta manera contribuir a la conservación del valor universal excepcional del bien y a la preservación de su integridad y autenticidad.

Para lograr todo esto la carta ha establecido siete principios fundamentales en los que la interpretación y presentación se deben basar:

- Acceso y comprensión. 
02.

\section{Centro de Interpretación CRTA en Durango.}

FOTO DE LA AUTORA

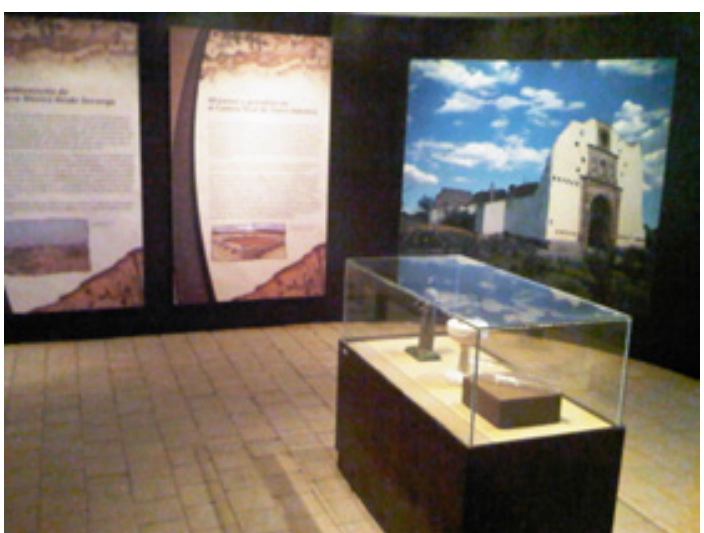

02.

- Fuentes de información.

- Atención al entorno y al contexto.

- Preservación de la autenticidad.

- Plan de sostenibilidad.

- Preocupación por la inclusión y la participación.

- Importancia de la investigación.

- Formación y evaluación.

La presentación e interpretación de un itinerario cultural como el Camino Real de Tierra Adentro deberá hacerse en varias etapas y con diferentes estrategias, de acuerdo a las necesidades de comunicación a las autoridades, a los habitantes (de todas la edades), a los propietarios de aquellos sitios que se encuentren en el régimen de propiedad privada, y a los visitantes y turistas.

No podemos negar que uno de los intereses de un sitio incluido en la lista del patrimonio mundial es el beneficio que puede dejar la actividad turística del bien, sobre todo en estos tiempos. El turismo se construye y prospera gracias a la existencia de atractivos culturales y naturales en buen estado de conservación y que cuentan con la infraestructura adecuada para recibir al visitante. Las festividades religiosas y civiles o la gastronomía son algunos ejemplos que motivan el viaje de un segmento particular de turistas.
Como también lo menciona la Carta Internacional sobre Turismo Cultural: «Un objetivo fundamental de la gestión del patrimonio consiste en comunicar su significado y la necesidad de su conservación tanto a la comunidad anfitriona como a los visitantes. El acceso físico, intelectual y/o emotivo, sensato y bien gestionado a los bienes del patrimonio, así como el acceso al desarrollo cultural, constituyen al mismo tiempo un derecho y un privilegio» (ICOMOS, 1999, p. 1). Sin embargo existen casos de bienes culturales o naturales que reciben visitantes sin tener las herramientas de interpretación y presentación adecuadas, lo que genera confusión o falta de información.

«Los programas para la protección y conservación del patrimonio natural y cultural en sus características físicas, en sus valores intangibles, expresiones culturales y sus variados contextos, deberían facilitar la comprensión y el aprecio de los significados de este patrimonio de un modo equilibrado y agradable» (ICOMOS, 1999, principio 1).

Recordemos que las amenazas para la preservación del patrimonio cultural no provienen del turismo en sí, sino de la errónea aplicación de las políticas de promoción, combinadas con la ausencia de reglamentos y planes de manejo para la canalización del turismo y el racional aprovechamiento de sus recursos. 


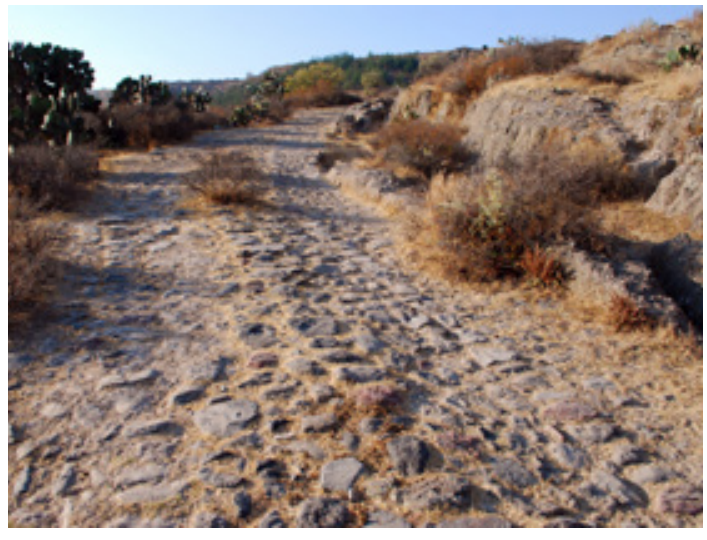

03.

Por su propia naturaleza, y como ámbito privilegiado, el turismo tendría que contribuir de forma especial al desarrollo sostenible, integrándose en el entorno natural, cultural y humano, y respetando los frágiles equilibrios que caracterizan a muchos destinos turísticos.

\section{DIFUSIÓN}

Las acciones de comunicación, interpretación y presentación de un itinerario cultural pueden ser partes integrantes de una comunicación planeada y ordenada en todas sus etapas que se conoce como plan de difusión y forma parte importante del plan de manejo que tiene por objetivo asegurar la protección efectiva del bien propuesto para el disfrute de las generaciones por venir.

Un plan de difusión es una herramienta eficaz e imprescindible para conocer, disfrutar, respetar, proteger y conservar los elementos que conforman el bien. Del mismo modo, el plan de difusión pretende generar conciencia en torno a los problemas ambientales que rodean al bien, y fomentar en la sociedad actitudes y valores de respeto y conservación de este entorno natural, además de hacer ver que es posible compatibilizar su explotación comercial con su preservación.
03. Tramo del CRTA.

FOTO: CORTESÍA INAH
Hablamos de explotación comercial porque es inevitable el interés que despierta la inclusión de un bien en la Lista del Patrimonio Mundial, ya sea por la promoción que se hace de la inclusión de un sitio, pero también por el hecho de poseer «valor universal excepcional, lo cual significa una importancia cultural y/o natural tan extraordinaria que trasciende las fronteras nacionales y cobra importancia para las generaciones presentes y venideras de toda la humanidad» (UNESCO, 2006).

El plan de difusión se debe estructurar teniendo en cuenta los principios fundamentales de la interpretación y presentación, para lograr las herramientas más adecuadas en cada una de las etapas de la difusión. Por lo cual también es necesario diseñar herramientas para los diferentes segmentos o auditorios a los que cada herramienta estará dirigida.

La etapa del plan de difusión considerada como interna contempla a la sociedad civil y las autoridades. Para su implementación se sugieren herramientas de comunicación -directas o cara a cara, y a través de canales como la tevé, la radio, medios impresos, etcétera)- enfocadas a los diferentes públicos (niños, jóvenes, adultos, empresarios, comerciantes, propietarios de inmuebles históricos, asociaciones civiles y religiosas, funcionarios y empleados de los diferentes niveles de gobierno). Las 
herramientas para niños y jóvenes se pueden desarrollar utilizando estrategias ya conocidas y probadas, como el programa de la UNESCO El Patrimonio Mundial en Manos de los Jóvenes. Conocerlo, Valorarlo y Actuar. También es importante sensibilizar mediante talleres dedicados a las autoridades responsables de la conservación, gestión y administración del bien, ya que no siempre se cuenta con personal especializado en esas áreas.

En materia de mercadotecnia y promoción, el reto del Camino Real de Tierra Adentro es informar al turista de su existencia, y de la oferta cultural y turística que ofrece, pero en términos reales, es decir, no invitar al turista si no se cuenta con la infraestructura necesaria para el disfrute del bien. Esta estrategia deberá servir además para crear conciencia en torno al turismo responsable y al manejo adecuado, para que contribuyan a su conservación.

El plan de mercadotecnia y promoción de un itinerario cultural es la segunda etapa de la difusión y forma parte del Plan de Desarrollo Turístico, que tendrá por objetivo potenciar la diversidad de la oferta turística con planes y oportunidades de negocios turísticos sustentables que fomenten el desarrollo y el beneficio para la sociedad.

\section{CONCLUSIÓN}

La comunicación, interpretación, presentación y difusión de los itinerarios culturales deben basarse en la comprensión del concepto y los aspectos específicos y significativos de cada itinerario. Al ser una categoría relativamente nueva y multidimensional, tenemos el reto de elaborar un plan de difusión que se incluya en el plan de manejo del bien, que será pionero en la manera de transmitir sus valores y contenidos a los diferentes receptores, en los 60 sitios que lo conforman.

La concientización sobre la importancia de la valoración y conservación de un bien debe hacerse con base en los documentos y normas internacionalmente reconocidas que indican claramente los pasos a seguir. Es igualmente importante considerar la legislación nacional y local.

Diseñar estrategias de acción en las cuales se integren métodos tradicionales de enseñanza y difusión con las nuevas herramientas disponibles debe y tiene que constituir un tema decisivo en la generación de una actitud de respeto y valoración hacia el patrimonio. Este aspecto es tan importante en un plan de manejo como lo puede ser el desarrollo sostenible del turismo o la restauración de inmuebles. 
Es muy diferente promocionar un puente o un tramo de un camino empedrado de hace cuatrocientos años, solamente porque es antiguo y está ahí. Al ser parte de un itinerario, ese puente probablemente fue construido por la necesidad de pasar un río en carreta o con una recua de mulas para transportar de manera segura productos y personas llegados de Europa y tal vez de África, que iban con destino a los centros mineros o a nuevas poblaciones, y esta carreta o recua volvería a pasar de regreso con un cargamento de plata y otros productos que se llevarían al viejo continente.

Los itinerarios culturales poseen una riqueza extraordinaria, por ser resultado y reflejo de los movimientos interactivos de personas, de intercambios continuos y recíprocos de bienes, ideas, conocimientos y valores dentro de un país o entre varios, y regiones a lo largo de considerables períodos, y haber generado una fecundación múltiple y recíproca de las culturas en el espacio y en el tiempo que se manifiesta en su patrimonio tangible e intangible.
Esta riqueza nos ofrece una posibilidad igualmente amplia para generar herramientas de difusión que despierten el interés no solo por conservar los sitios sino por conocer más sobre las razones que motivaron la construcción de cada uno de ellos, que ahora son parte del Camino Real de Tierra Adentro. Más aun, nos da la oportunidad de rescatar aspectos olvidados de los intercambios que se dieron a lo largo de él y que hoy nos muestran resultados que tal vez no hemos observado detenidamente pero que gracias a la presentación e interpretación de este itinerario veremos con otros ojos.

RECIBIDO: 21 de octubre de 2014 ACEPTADO: 5 de diciembre de 2014 


\section{BIBLIOGRAFÍA}

ACOSTA, R. M. (2010). «Metodología aplicada a la protección de itinerarios culturales, con especial atención hacia aquellos incluidos en la Lista del Patrimonio Mundial». CIIC ICOMOS, Madrid, España.

- (2011). Carpeta Informativa sobre la Inscripción del Centro Histórico de la Ciudad de San Luis Potosí en el Camino Real de Tierra Adentro, elaborada por la autora en y para la Secretaría de Cultura de San Luis Potosí, México.

BALLART, J. y J. TRESSERRAS (2005). Gestión del patrimonio cultural. Barcelona: Ariel.

CAMPESINO Fernández, A. J. (2001). «Centros Históricos Latinoamericanos, Patrimonio de la Humanidad: Planificación, Gestión y Seguimiento Efectivo de su Conservación». Seminario Internacional de Ciudades Históricas Iberoamericanas.

CENTRO DEL PATRIMONIO MUNDIAL - UNESCO (2008). «Directrices prácticas sobre la aplicación de la Convención para la Protección del Patrimonio Mundial. Comité Intergubernamental de Protección del Patrimonio Mundial Cultural y Natural». Centro del Patrimonio Mundial.

CONACULTA (2006). Cuadernos de Patrimonio Culturaly Turismo $\mathrm{N}^{\circ} 15$. México.

ICOMOS (1999). «Carta Internacional sobre Turismo Cultural. La Gestión del Turismo en los sitios con Patrimonio Significativo». Adoptada por ICOMOS en la $12^{\mathrm{a}}$ Asamblea General en México.

- (2008). «Carta de Itinerarios Culturales». Elaborada por el Comité Científico Internacional de Itinerarios Culturales (CIIC) del ICOMOS. Ratificada por la $16^{\text {a }}$ Asamblea General del ICOMOS, Quebec (Canadá).
- (2008, b). «Carta para Interpretación y Presentación de Sitios de Patrimonio Cultural». Preparado bajo los auspicios del Comité Científico Internacional del ICOMOS sobre la Interpretación y Presentación de Sitios de Patrimonio Cultural. Ratificada por la $16^{\mathrm{a}}$ Asamblea General del ICOMOS, Quebec (Canadá).

ICOMOS México (2002). «Carta de Pátzcuaro». XXII Symposium Internacional de Conservación del Patrimonio Monumental. Pátzcuaro, Michoacán.

INAH (2009). Expediente de Nominación del Camino Real de Tierra Adentro.

PADILLA, M. (2010). Comunicación y Patrimonio. Cuba: Oficina del Historiador de la Ciudad de Camagüey.

PEDERSEN, A. (2002). Managing Tourism at World Heritage Sites: a Practical Manual for World Heritage Site Managers. UNESCO World Heritage Centre.

RINGBECK B. (2008). Management Plans for World Heritage Sites. A Practical Guide. German Commission for UNESCO

UNESCO (1972). Convención sobre la Protección del Patrimonio Mundial, Cultural y Natural. Adoptada por la Conferencia General de la UNESCO, en su 17a, reunión celebrada en París.

- (2001). Declaración Universal sobre la Diversidad Cultural. Adoptada por la 31 reunión de la Conferencia General de la UNESCO. París.

- (2003). Convención para la Salvaguardia del Patrimonio Cultural Inmaterial, París, Francia.

- (2006). Directrices Prácticas para la aplicación de la Convención del Patrimonio Mundial. 